$01.1 ; 08.3$

\title{
Применение метода матрицы рассеяния для расчета примесных состояний в полупроводниковых структурах
}

\author{
(C) C.B. Морозов ${ }^{1,2}$, М.C. Жолудев ${ }^{1,2}$ \\ ${ }^{1}$ Институт фризики микроструктур РАН, Нижний Новгород, Россия \\ ${ }^{2}$ Нижегородский государственный университет им. Н.И. Лобачевского, Нижний Новгород, Россия \\ E-mail: more@ipmras.ru
}

Поступило в Редакцию 18 декабря 2020г.

В окончательной редакции 18 декабря 2020 г.

Принято к публикации 21 декабря 2020г.

\begin{abstract}
Метод матрицы рассеяния адаптирован для расчета уровней энергии и волновых функций носителей заряда вблизи примесно-дефектных центров. Возможность применения данного метода для многозонных моделей продемонстрирована на примере гамильтониана Латтинжера с кулоновским акцептором в приближении сферической симметрии. Полученные значения энергии дискретных уровней хорошо согласуются с результатами расчетов, выполненных другими методами.
\end{abstract}

Ключевые слова: $\mathrm{HgCdTe}$, примесь, узкозонные полупроводники, матрица рассеяния.

DOI: 10.21883/PJTF.2021.07.50795.18663

Значительные успехи в получении длинноволнового стимулированного излучения в узкозонных гетероструктурах с квантовыми ямами $\mathrm{Hg}_{1-x} \mathrm{Cd}_{x} \mathrm{Te} / \mathrm{Cd}_{y} \mathrm{Hg}_{1-y} \mathrm{Te}$ [1] делают актуальными задачи создания $p-n$-перехода и изучения влияния примесей на процессы безызлучательной рекомбинации в образцах такого типа. Это в свою очередь требует развития методов численного моделирования примесных состояний в узкозонных полупроводниках, особенностью изучения которых является необходимость учитывать большое количество близких по энергии зон при теоретических расчетах. Вследствие этого при численном решении уравнения Шредингера возникает сильная неустойчивость, связанная с экспоненциально растущими решениями, которая проявляется не только в запрещенной зоне, но и в области непрерывного спектра (см., например, работу [2]). Здесь представляется перспективным применение метода матрицы рассеяния [3-6], который обладает высокой численной устойчивостью. Ранее в системах с плавным потенциалом такой метод использовался только для задач с одним уравнением, что применительно к полупроводникам соответствует приближению эффективной массы. В настоящей работе мы предлагаем обобщение данного подхода для многозонного приближения. В качестве примера применения обобщенного метода рассмотрим модель Латтинжера с кулоновским акцептором в приближении сферической симметрии [7].

В рамках приближения огибающих функций [8] с учетом сферической симметрии $[2,7,9,10]$ волновая функция электрона записывается следующим образом:

$$
\Psi_{\lambda}^{(J, M)}(\mathbf{r})=\sum_{n=1}^{n_{f}} f_{\lambda, n}^{(J)}(r) \Phi_{\lambda, n}^{(J, M)}(\mathbf{r})
$$

где $\Phi_{\lambda, n}^{(J, M)}(\mathbf{r})$ - собственные функции оператора квадрата полного углового момента (собственное значение $J$ ) и его проекции на ось $z$ (собственное значение $M$ ), которые включают в себя угловую зависимость и базис Кона-Латтинжера. Индекс $\lambda$ содержит все остальные квантовые числа, набор которых, как и конкретный вид функций $\Phi_{\lambda, n}^{(J, M)}(\mathbf{r})$, зависит от рассматриваемой модели. Таким образом, волновая функция вида (1) определяется вектором функций $\mathbf{f}_{\lambda}^{(J)}(r)$ размерности $n_{f}$, который удовлетворяет уравнению Шредингера с матричным гамильтонианом

$$
\left[\hat{\mathbf{H}}_{0}(J, \lambda)+V(r)\right] \mathbf{f}=E \mathbf{f},
$$

где $\hat{\mathbf{H}}_{0}(J, \lambda)$ - гамильтониан электрона в однородном полупроводнике, а $V(r)$ - потенциал примеси. Далее для наглядности опустим параметры $J$ и $\lambda$.

Применение метода матрицы рассеяния для решения уравнения (2) вблизи $r=0$ в общем случае не представляется возможным, поскольку в этой точке потенциал примеси, как правило, имеет особенность. Выберем близкую к нулю точку $r_{0}$, такую, что при $r \leqslant r_{0}$ неустойчивость не мешает получить решение уравнения (2) методом Рунге-Кутта. Выберем также точку $r_{N}$, достаточно далекую от нуля, чтобы при $r \geqslant r_{N}$ мы могли считать потенциал примеси равным нулю. В области $\left[r_{0}, r_{N}\right]$ будем искать решение уравнения (2) с помощью метода матрицы рассеяния. Для этого разобьем ее на отрезки серией точек $r_{0}, r_{1}, \ldots, r_{N}$. На каждом отрезке $\left[r_{j}, r_{j+1}\right]$ будем считать потенциал постоянным и равным $V_{j}=\left[V\left(r_{j}\right)+V\left(r_{j+1}\right)\right] / 2$. Тогда уравнение (2) примет вид

$$
\hat{\mathbf{H}}_{0} \mathbf{f}_{j}=\left(E-V_{j}\right) \mathbf{f}_{j} .
$$

Выражение (3) представляет собой систему из $n_{f}$ уравнений второго порядка, которая имеет $2 n_{f}$ частных решений, представляющих собой наборы сфериче- 
ских волн

$$
\mathbf{f}_{j, n}^{(a, b)}(r)=\left(\begin{array}{c}
c_{j, n, 1} S_{L_{1}, \pm k_{j, n}}(r) \\
\vdots \\
c_{j, n, n_{f}} s_{L_{n_{f}}, \pm k_{j, n}}(r)
\end{array}\right), n=1, \ldots, n_{f},
$$

где $s_{L, k}(r)=j_{L}(k r)+i y_{L}(k r)$, а $j_{L}$ и $y_{L}-$ сферические функции Бесселя первого и второго рода соответственно. Отметим, что эти функции определены как для вещественных, так и для мнимых значений $k_{j, n}$ (см., например, [11]). Мы можем считать, что в выражении (4) $k_{j, n}$ является положительным вещественным числом либо мнимым числом с положительной мнимой частью. Тогда функции $\mathbf{f}_{j, n}^{(a)}(r)$ будут сферическими волнами, бегущими от центра (для вещественных $k_{j, n}$ ), либо экспоненциально затухающими решениями (для мнимых $\left.k_{j, n}\right)$. В этом случае функции $\mathbf{f}_{j, n}^{(b)}(r)$, имеющие противоположный знак волнового числа, будут соответственно сферическими волнами, бегущими к центру, либо экспоненциально растущими решениями. Такая классификация решений обеспечивает устойчивость метода матрицы рассеяния [3]. Значения волнового числа $k_{j, n}$ и коэффициенты $c_{j, n, i}$ получаются путем подстановки выражения (4) в уравнение (3).

Приближенное решение уравнения (2) на отрезке $\left[r_{j}, r_{j+1}\right]$ будет линейной комбинацией всех частных решений (4):

$$
\mathbf{f}_{j}(r) \simeq \mathbf{F}_{j}^{(a)}(r) \mathbf{a}_{j}+\mathbf{F}_{j}^{(b)}(r) \mathbf{b}_{j}
$$

где матрицы $\mathbf{F}_{j}^{(a, b)}(r)$ составлены из столбцов вида (4):

$$
\mathbf{F}_{j}^{(a, b)}(r)=\left(\mathbf{f}_{j, 1}^{(a, b)}(r), \ldots, \mathbf{f}_{j, n_{f}}^{(a, b)}(r)\right),
$$

a неизвестные векторы $\mathbf{a}_{j}$ и $\mathbf{b}_{j}$ должны обеспечить непрерывность и гладкость функции $\mathbf{f}_{j}(r)$ на границах отрезков. Следуя процедуре, подробно описанной в работе [3], получим набор матриц рассеяния, удовлетворяющих следующему выражению:

$$
\left(\begin{array}{l}
\mathbf{a}_{j_{2}} \\
\mathbf{b}_{j_{1}}
\end{array}\right)=\left(\begin{array}{ll}
\mathbf{S}_{j_{1}, j_{2}}^{(a a)} & \mathbf{S}_{j_{1}, j_{2}}^{(a b)} \\
\mathbf{S}_{j_{1}, j_{2}}^{(b a)} & \mathbf{S}_{j_{1}, j_{2}}^{(b b)}
\end{array}\right)\left(\begin{array}{l}
\mathbf{a}_{j_{1}} \\
\mathbf{b}_{j_{2}}
\end{array}\right), \quad j_{1}<j_{2} .
$$

Чтобы использовать это выражение для вычисления векторов $\mathbf{a}_{j}$ и $\mathbf{b}_{j}$, нужно знать значения $\mathbf{a}_{0}$ и $\mathbf{b}_{N}$ (см. [3]).

Из условия конечности волновой функции при $r \rightarrow \infty$ следует, что все компоненты вектора $\mathbf{b}_{N}$, соответствующие экспоненциальным решениям, должны быть равны нулю. В области непрерывного спектра оставшиеся ненулевые компоненты $\mathbf{b}_{N}$ вычисляются из условий нормировки и ортогональности волновых функций. В области дискретного спектра имеем $\mathbf{b}_{N}=0$. Таким образом, мы можем считать вектор $\mathbf{b}_{N}$.

Для вычисления вектора $\mathbf{a}_{0}$ аналогично работе [2] c помощью обобщенного метода Фробениуса [12] и метода Рунге-Кутта в области $r \leqslant r_{0}$ найдем $n_{f}$ линейно независимых решений уравнения (2), которые не имеют особенности в точке $r=0$ и образуют матрицу $\mathbf{F}_{-1}^{(a)}(r)$ (мы используем индекс -1 , поскольку эти решения находятся левее отрезка с индексом 0). Из условия непрерывности и гладкости решения в точке $r_{0}$ получим следующее выражение:

$$
\left(\begin{array}{l}
\mathbf{a}_{0} \\
\mathbf{b}_{0}
\end{array}\right)=\left(\begin{array}{l}
\mathbf{T}^{(a)} \\
\mathbf{T}^{(b)}
\end{array}\right) \mathbf{a}_{-1}
$$

где

$$
\left(\begin{array}{c}
\mathbf{T}^{(a)} \\
\mathbf{T}^{(b)}
\end{array}\right)=\left(\begin{array}{cc}
\mathbf{F}_{0}^{(a)}\left(r_{0}\right) & \mathbf{F}_{0}^{(b)}\left(r_{0}\right) \\
\mathbf{F}_{0}^{\prime(a)}\left(r_{0}\right) & \mathbf{F}_{0}^{\prime(b)}\left(r_{0}\right)
\end{array}\right)^{-1}\left(\begin{array}{c}
\mathbf{F}_{-1}^{(a)}\left(r_{0}\right) \\
\mathbf{F}_{-1}^{\prime(a)}\left(r_{0}\right)
\end{array}\right)
$$

Подставив в формулу (5) значения $j_{1}=0, j_{2}=N$ и выражение (6) для $\mathbf{a}_{0}$ и $\mathbf{b}_{0}$, получаем следующее уравнение:

$$
\mathbf{Q}_{-1}=\mathbf{S}_{0, N}^{(b b)} \mathbf{b}_{N}
$$

где

$$
\mathbf{Q}=\mathbf{T}^{(b)}-\mathbf{S}_{0, N}^{(b a)} \mathbf{T}^{(a)} .
$$

Поскольку для локализованных состояний $\mathbf{b}_{N}=0$, условием для энергии уровней дискретного спектра будет

$$
\operatorname{det} \mathbf{Q}(E)=0 .
$$

В качестве примеров применения данного метода рассмотрим две системы, решения для которых были найдены другими способами: водородоподобный центр и кулоновский акцептор в сферически-симметричной модели Латтинжера [7].

Для водородоподобного центра выражение для волновой функции электрона (1) выглядит следующим образом:

$$
\Psi_{L}^{(J, M)}(\mathbf{r})=f_{L}^{(J)}(r) \Phi_{L}^{(J, M)}(\mathbf{r})
$$

где $L=J \pm 1 / 2$. Размерность решения равна единице $\left(n_{f}=1\right)$, а гамильтониан однородного полупроводника и потенциал в уравнении (2) равны

$$
\begin{gathered}
\hat{\mathbf{H}}_{0}(J, L)=-\frac{d^{2}}{d r^{2}}-\frac{2}{r} \frac{d}{d r}+\frac{L(L+1)}{r^{2}}, \\
V(r)=-\frac{2}{r},
\end{gathered}
$$

где в качестве единиц измерения длины и энергии выбраны соответственно боровский радиус $\left(a_{0}=\hbar^{2} / m e^{2}\right)$ и ридберг $\left(E_{0}=m e^{4} / 2 \hbar^{2}\right)$. Точное решение этой задачи известно, и значения энергии дискретных уровней равны [6]:

$$
E_{L, n}=\frac{1}{(n+L)^{2}}, \quad n=1,2, \ldots
$$

Эти энергии, так же как результаты расчетов функции $\operatorname{det} \mathbf{Q}(E)$ в уравнении (7), приведены на рис. 1, из которого хорошо видно, что действительная и мнимая части $\operatorname{det} \mathbf{Q}(E)$ одновременно обращаются в нуль именно на уровнях дискретного спектра. Отметим, что мы 
получили тот же результат, что и в работе [6]. При этом вместо плоских волн мы использовали сферические волны, что позволило обойтись без замены искомой функции в уравнении (2) и не включать в потенциал слагаемые, зависящие от $L$.

Теперь рассмотрим кулоновский акцептор в сферически-симметричной модели Латтинжера [7]. По аналогии с работами $[2,7,10]$ мы можем представить волновую функцию электрона в такой системе в виде

$$
\Psi_{L}^{(J, M)}(\mathbf{r})=f_{L+1}^{(J)}(r) \phi_{L+1,3 / 2}^{(J, M)}(\mathbf{r})+f_{L-1}^{(J)}(r) \phi_{L-1,3 / 2}^{(J, M)}(\mathbf{r}),
$$

где $L=J \pm 1 / 2$, а $\phi_{L, 3 / 2}^{(J, M)}-$ собственные функции оператора квадрата полного углового момента и его проекции на ось $z$ (собственные числа $J$ и $M$ ), полученные из состояний с орбитальным моментом $L$ и спином 3/2. Размерность решения равна двум $\left(n_{f}=2\right)$, а гамильтониан однородного полупроводника и потенциал будут теми же, что и в работе [7].

В зависимости от минимального значения орбитального момента в формуле (8) состояния акцептора делятся на уровни $S$-типа, $P$-типа и т.д. [7]. Отметим, что в случае $J=1 / 2$ в выражении (8) сохраняется только первое слагаемое, и мы получаем два одномерных двукратно вырожденных решения $P$ - и $D$-типа.

На рис. 2 приведены полученные различными методами зависимости энергий дискретных уровней $S$ - и $P$-типа от параметра $\mu$, который определяет отношение масс легких и тяжелых дырок [7]: $m_{l h} / m_{h h}=(1-\mu) /(1+\mu)$. В настоящей работе результаты были получены путем решения уравнения (7), а в работе [7] — с помощью вариационного метода. Хорошо видно, что результаты

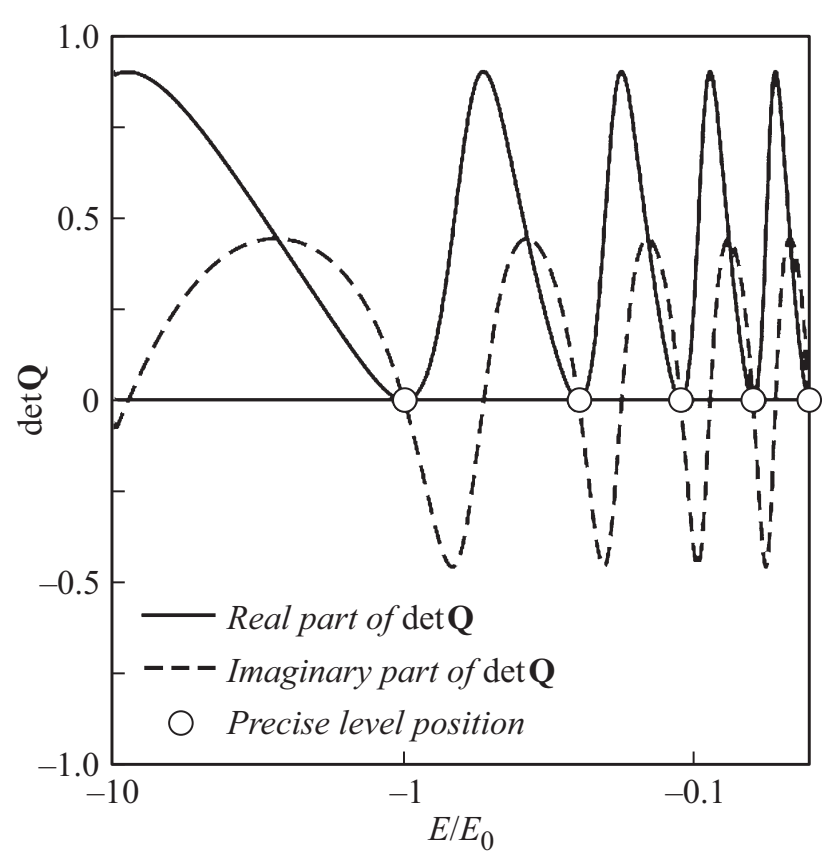

Рис. 1. Рассчитанная зависимость величины $\operatorname{det} \mathbf{Q}$ от энергии в области дискретного спектра водородоподобного центра при $L=0$. Масштаб по оси энергий логарифмический.

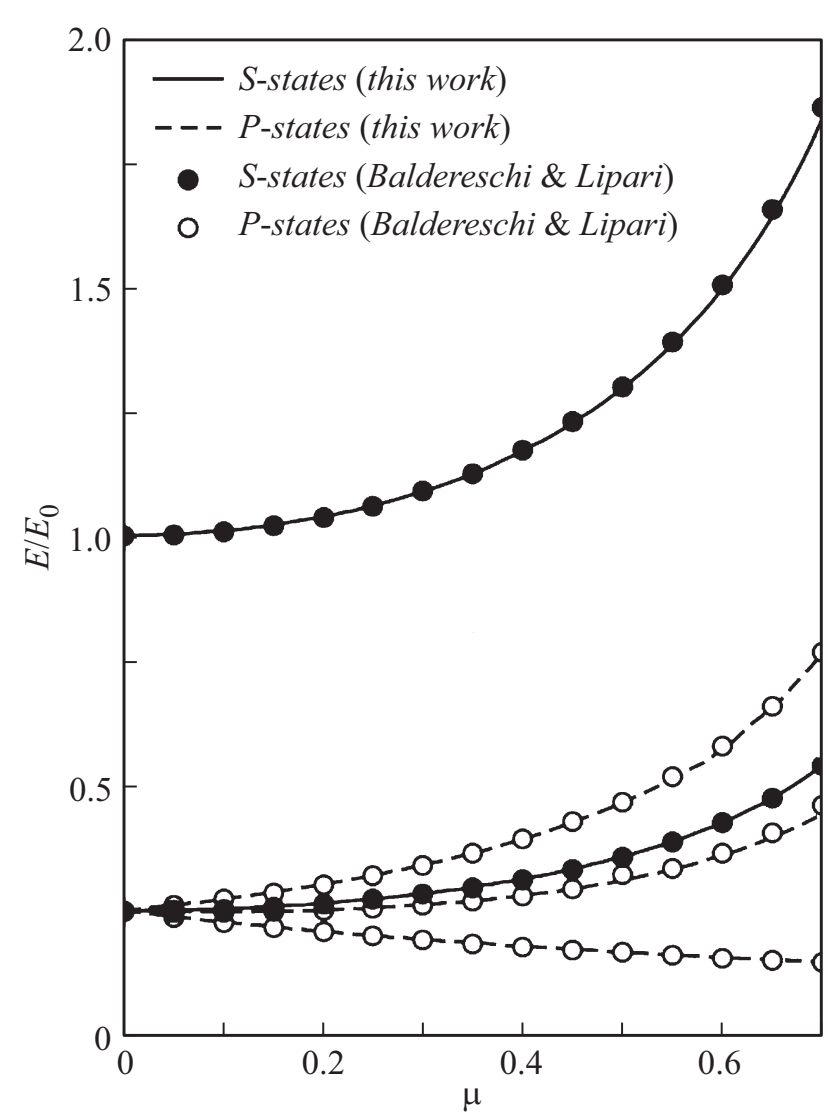

Рис. 2. Результаты расчетов зависимости энергий дискретных уровней кулоновского акцептора от параметра $\mu$.

расчетов дискретного спектра, полученные разными способами, практически совпадают.

Метод матрицы рассеяния уже достаточно давно применяется для решения задач как со ступенчатым, так и с плавно меняющимся потенциалом. Он хорошо зарекомендовал себя благодаря своей численной устойчивости. В настоящей работе мы провели обобщение этого метода на случай систем дифференциальных уравнений произвольной размерности. Применение обобщенного метода к задаче об акцепторе в сферическисимметричной модели Латтинжера продемонстрировало хорошее согласие с полученными ранее теоретическими результатами. Аналогичный подход может использоваться для изучения локализованных и резонансных примесных состояний в полупроводниковых структурах на основе узкозонных и бесщелевых материалов (включая соединения $\left.\mathrm{Cd}_{x} \mathrm{Hg}_{1-x} \mathrm{Te}\right)$.

\section{Финансирование работы}

Работа выполнена при поддержке Министерства науки и высшего образования РФ (грант № 075-15-2020-797 (13.1902.21.0024)). 


\section{Конфликт интересов}

Авторы заявляют, что у них нет конфликта интересов.

\section{Список литературы}

[1] S. Morozov, V. Rumyantsev, M. Fadeev, M. Zholudev, K. Kudryavtsev, A. Antonov, A. Kadykov, A. Dubinov, N. Mikhailov, S. Dvoretskii, V. Gavrilenko, Appl. Phys. Lett., 111, 192101 (2017). DOI: 10.1063/1.4996966

[2] М.С. Жолудев, Д.В. Козлов, Н.С. Куликов, А.А. Разова, В.И. Гавриленко, С.В. Морозов, ФТП, 54 (8), 695 (2020). DOI: 10.21883/FTP.2020.08.49652.9404

[3] D.Y.K. Ko, J.C. Inkson, Phys. Rev. B, 38, 9945 (1988). DOI: 10.1103/PhysRevB.38.9945

[4] M.S. De Bianchi, M.D. Ventra, Eur. J. Phys., 16, 260 (1995). DOI: 10.1088/0143-0807/16/6/003

[5] C. Ramirez, R. Leon, J. Phys. Soc. Jpn., 86, 114002 (2017). DOI: 10.7566/JPSJ.86.114002

[6] C. Ramirez, F.H. Gonzalez, C.G. Galvan, J. Phys. Soc. Jpn., 88, 094002 (2019). DOI: 10.7566/JPSJ.88.094002

[7] A. Baldereschi, N.O. Lipari, Phys. Rev. B, 8, 2697 (1973). DOI: 10.1103/PhysRevB.8.2697

[8] J.M. Luttinger, W. Kohn, Phys. Rev., 97, 869 (1955). DOI: 10.1103/PhysRev.97.869

[9] Д.А. Варшалович, А.Н. Москалев, В.К. Херсонский, Квантовая теория углового момента (Наука, Л., 1975).

[10] E.P. Pokatilov, V.A. Fonoberov, V.M. Fomin, J.T. Devreese, Phys. Rev. B, 64, 245328 (2001). DOI: 10.1103/PhysRevB.64.245328

[11] М. Абрамовиц, И. Стиган, Справочник по специальным бункциям (Наука, М., 1979).

[12] M. Barkatou, T. Cluzeau, C. El Bacha, in Proc. of mathematical theory of networks and systems (Budapest, Hungary, 2010), p. 1059. 